\section{WHAT IS NSW HEALTH DOING ABOUT SALMONELLOSIS?}

NSW Health works with other state and national organisations on strategies such as:

- $\quad$ surveying food types across the states. High-risk foods are surveyed for the presence of bacteria such as Salmonella. Findings are reported to the government and the food industry to improve food standards;

- monitoring frequency of salmonellosis and investigating cases to determine the cause of infection;
- intervening to stop the spread of salmonellosis: for example, correcting food preparation practice in kitchens; public education (for example, fact sheets); withdrawing infected foods from the market; and auditing food outlets.

For further information please contact your local public health unit, community health centre, or doctor.

May 2002 g

\title{
COMMUNICABLE DISEASES, NSW: MAY 2002
}

\section{TRENDS}

The first quarter of 2002 saw relatively few notifications of people diagnosed with arbovirus infections, meningococcal disease, and shigellosis in NSW (Figure 1). The epidemic of pertussis continued to decline throughout the state. The increase in hepatitis A identified in December 2001 in Sydney among men-who-have-sexwith-men did not continue, with notifications of hepatitis A subsequently declining (20 cases notifications were received in March, Table 1). On the other hand, notifications of cryptosporidiosis remained relatively high in the first quarter of 2002, including 57 cases that were notified in March. Most cases are from rural areas, and children under five years of age are disproportionately affected.

\section{MEASLES CASES REPORTED}

Two cases of measles were reported in April, ending a five-month measles-free period in NSW. Both cases became infected while travelling overseas. The South Eastern, South Western, and Central Sydney Public Health Units investigated both cases.

Case 1 is a 23-year-old woman who travelled to Bali in March. After returning to Australia, she developed a fever, aches and pains, and headache, followed by a cough and sore throat. A rash appeared five days later. The rash began on the face and then spread to the neck, chest, arms, and legs. Case 1 made four visits to medical clinics before the rash appeared and the diagnosis was made. Case 1 had no clear history of measles vaccination, but states she had received her usual vaccines as a baby. Serology was taken four days after the rash first appeared and tested positive for measles IgM.

Case 2 is an 11-month-old girl who travelled in Asia until late March. While travelling, she developed some intermittent diarrhoea and a runny nose. In early April she developed a fever, anorexia, and was miserable. She developed a cough and a rash began on her forehead and back of neck. The rash spread to her face, neck, trunk, and limbs and she developed conjunctivitis. Case 2 made several visits to medical facilities before the rash appeared and diagnosis was made. Case 2 had not been vaccinated against measles. Serology and nasopharyngeal aspirates were taken in early April. The serum tested positive for measles $\operatorname{IgM}$ and the nasopharyngeal aspirate tested positive for measles on immuno-fluorescence testing.

The cases highlight two important public health messages:

- Measles is now rare in Australia but remains common overseas. The MMR vaccine (that protects against measles, mumps and rubella) should now be considered to be part of the overseas travellers' routine pre-travel health check, especially for anyone born after 1970.

- Because it is rare, measles is not often considered as a diagnosis by health care workers. The diagnosis should be considered in patients presenting with a rash-illness that includes cough, and fever at the onset of the rash, or if there is a history of possible exposure to measles (for example, contact with a suspected case or travel to an endemic area in the 7-18 days before onset of fever). Confirmation of the diagnosis with serology 
(measles IgM) is essential. Early in the illness, viral cultures can be grown from blood samples, conjunctival swabs, nasopharyngeal swabs or aspirates, and even urine, and can help in the diagnosis. Doctors and laboratories must report all suspected cases by telephone to their local public health unit.

Individuals with measles are infectious from just before the onset of the symptoms (up to five days before onset of the rash) until four days after the rash appears. In clinical settings (such as waiting rooms), patients with suspected measles must be isolated from other patients, and the area in which they waited should be kept clear of susceptible patients for at least two hours after the infectious person has left.

\section{ENHANCED INFLUENZA SURVEILLANCE BEGINS}

From late April, the NSW Department of Health has coordinated enhanced influenza surveillance. This will identify trends in influenza infections in NSW, and help characterise the strains of influenza prevalent in the community. As in 2001, surveillance brings together several data sources for weekly reporting. These sources are:
- routine laboratory notifications of confirmed influenza infections;

- enhanced laboratory surveillance from six major laboratories in Sydney and the Hunter Areas for respiratory virus testing and their results;

- sentinel surveillance around the state for influenzalike illness, in patients presenting to selected general practitioners each week;

- directed virological surveillance, where selected general practitioners take respiratory samples for viral testing from patients they suspect to have influenza.

Influenza can cause a serious illness, especially in the elderly, or people with heart, lung or metabolic diseases, many of whom will require hospitalisation and some of whom may even die this winter as a result of their infection. People wishing to avoid influenza, especially those at high risk for complications, should be vaccinated each year with the influenza vaccine well before winter begins. In 2002, the influenza vaccine is designed to protect against three strains of influenza that are likely to be circulating in Australia. These are the H1N1 (A/New Caledonia/20/99), the H3N2 (A/Moscow/10/99) and the

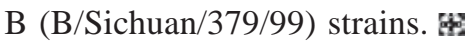




\section{FIGURE 1}

\section{REPORTS OF SELECTED COMMUNICABLE DISEASES, NSW, JANUARY 1997 TO MARCH 2002,}

BY MONTH OF ONSET

These are preliminary data: case counts for recent months may increase because of reporting delays. Laboratory-confirmed cases, except for measles, meningococcal disease and pertussis _ actual _ _ _ predicted after adjusting for likely reporting delays.

\begin{tabular}{|rc|}
\hline \multicolumn{2}{|c|}{ NSW population } \\
Male & $50 \%$ \\
$<5$ & $7 \%$ \\
$5-24$ & $28 \%$ \\
$25-64$ & $52 \%$ \\
$65+$ & $13 \%$ \\
Rural $^{*}$ & $42 \%$ \\
\hline
\end{tabular}
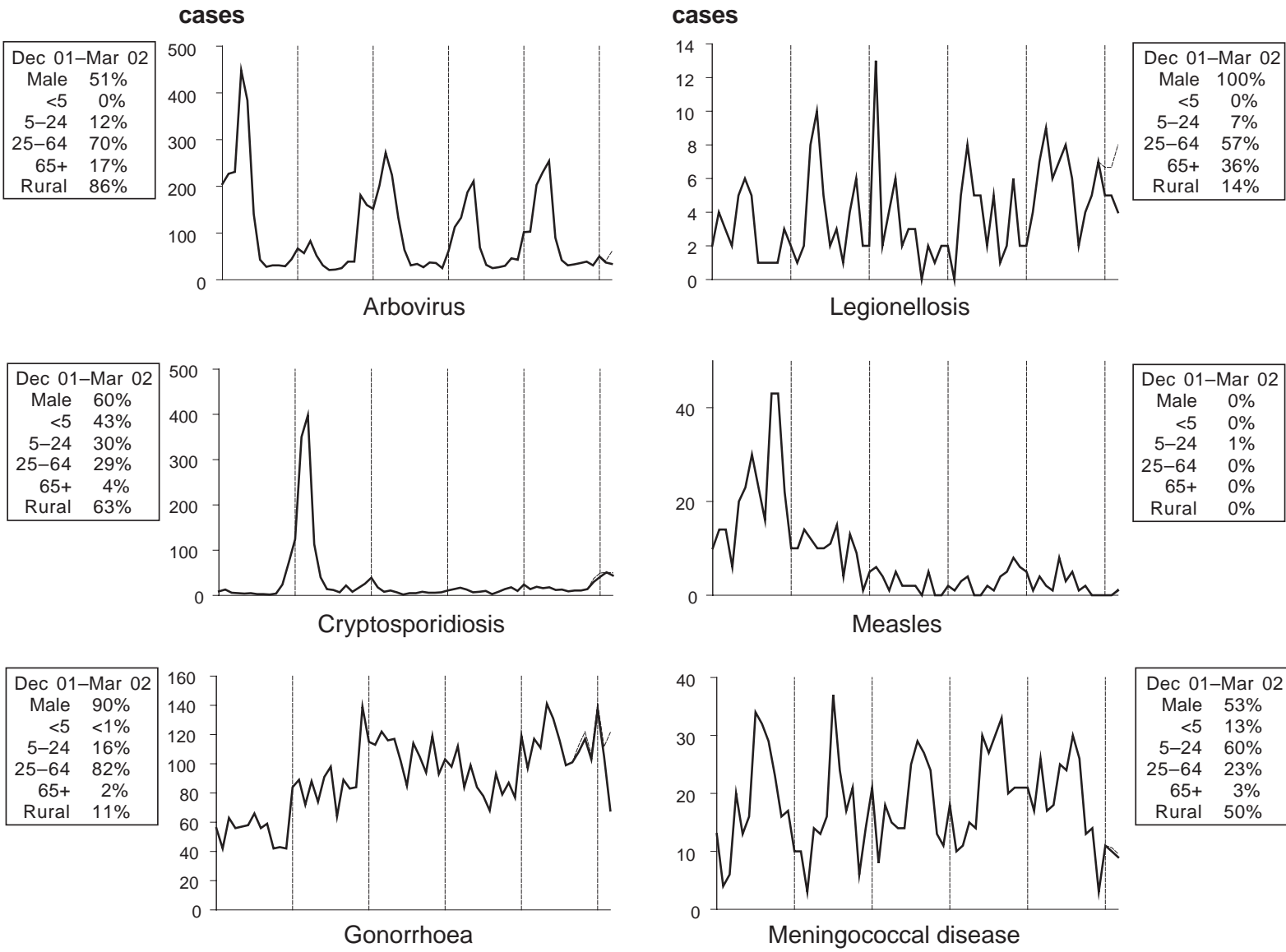

Dec 01-Mar 02 Male $53 \%$ $<5 \quad 13 \%$ $5-24 \quad 60 \%$ \begin{tabular}{rr}
-64 & -2 \\
$65+$ & $3 \%$ \\
\hline
\end{tabular} Rural $50 \%$

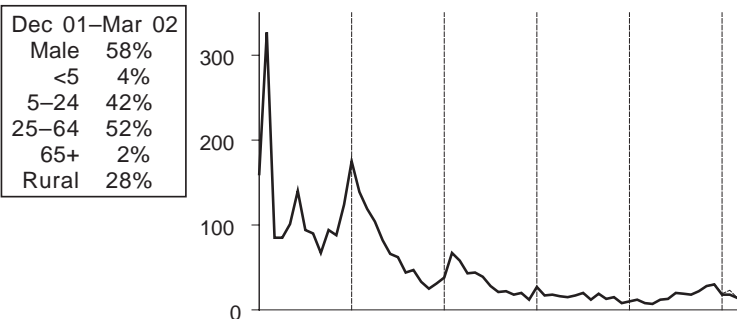

Hepatitis A

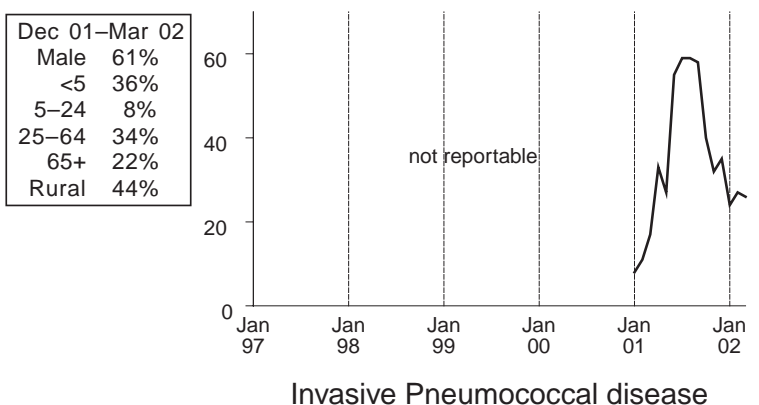

For definition, see NSW Public Health Bulletin, April 2000

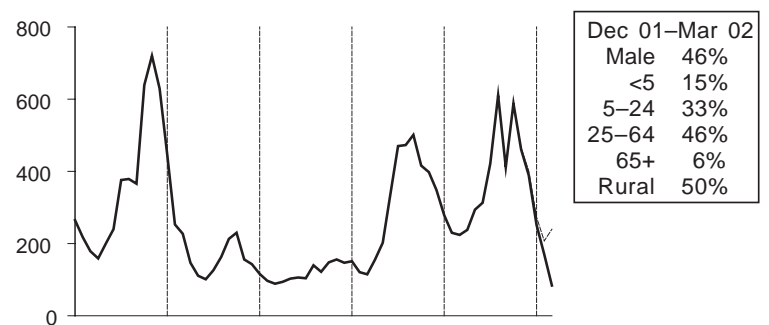

Pertussis

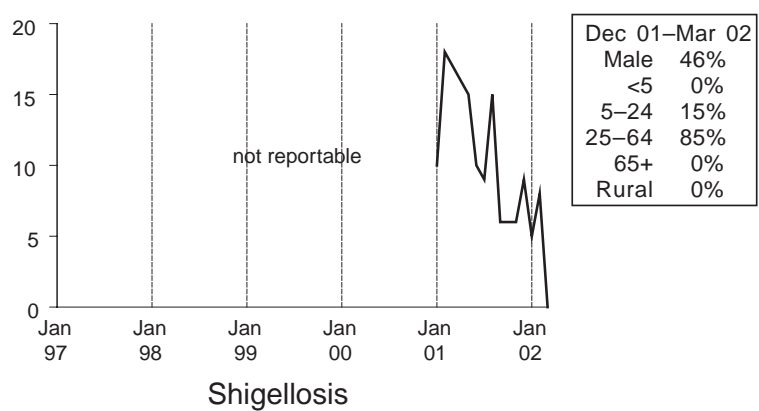




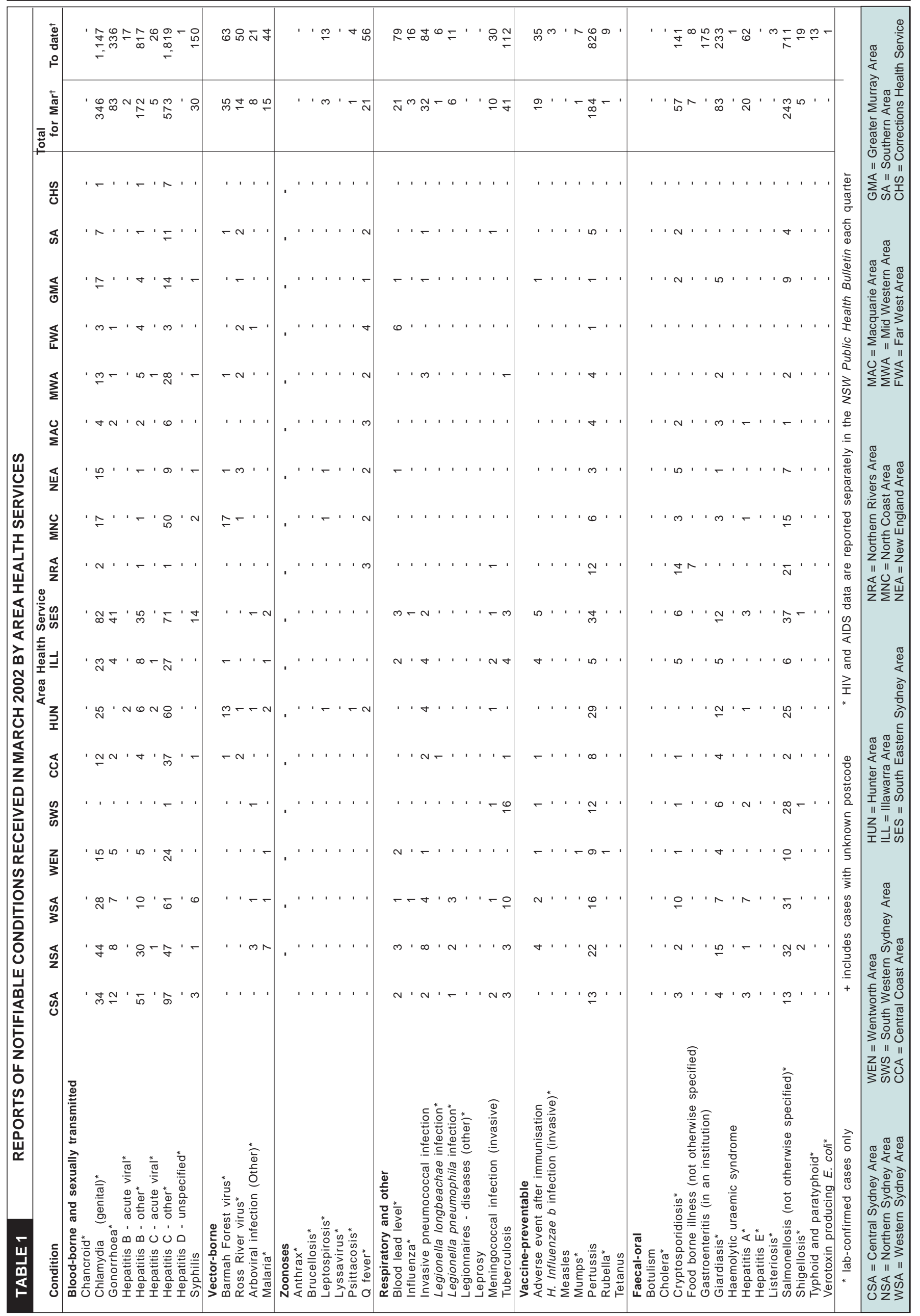

